\title{
COMPUTATION OF SOME SECOND ORDER STURM- LIOUVILLE BVPS USING CHEBYSHEV-LEGENDRE COLLOCATION METHOD
}

\author{
Humaira Farzana ${ }^{1}$ and Md. Shafiqul Islam ${ }^{2}$ \\ ${ }^{1}$ Department of Arts \& Sciences, Ahsanullah University of Science \& Technology \\ Dhaka-1215, Bangladesh. \\ ${ }^{2}$ Department of Applied Mathematics, Dhaka University, Dhaka-1000, Bangladesh \\ Corresponding author: humaira_tithy@yahoo.com
}

Received 22.03.2015

Accepted 18.08.2015

\begin{abstract}
We propose Chebyshev-Legendre spectral collocation method for solving second order linear and nonlinear eigenvalue problems exploiting Legendre derivative matrix. The Sturm-Liouville (SLP) problems are formulated utilizing Chebyshev-Gauss-Lobatto (CGL) nodes instead of Legendre Gauss-Lobatto (LGL) nodes and Legendre polynomials are taken as basis function. We discuss, in details, the formulations of the present method for the Sturm-Liouville problems (SLP) with Dirichlet and mixed type boundary conditions. The accuracy of this method is demonstrated by computing eigenvalues of three regular and two singular SLP's. Nonlinear Bratu type problem is also tested in this article. The numerical results are in good agreement with the other available relevant studies.
\end{abstract}

Keywords: Sturm-Liouville problems, Eigenvalue, Chebyshev nodes, Legendre polynomials, Spectral collocation method

\section{Introduction}

Spectral methods namely spectral Galerkin, spectral collocation, spectral Tao methods etc. are extensively used in the field of applied sciences and engineering due to the better performance and exponentially rapid convergent rate in preference to algebraic convergence rates for finite difference and finite element methods. Many researchers contributed to their works to the study of spectral Chebyshev collocation method for computing eigenvalues of second order SturmLiouville problems. Not much works are found for the solution of SLP's applying spectral collocation method using Legendre derivative matrix in the recent years. In this paper, we present spectral collocation method that offers accurate solutions which are put up with in terms of truncated series of smooth polynomial functions.

For the solutions of SLP's some studies are carried out by various numerical schemes. Min and Gottlieb [4] applied domain decomposition techniques for spectral methods. To obtain the accuracy, the authors classified each subdomain by the finite degrees of Chebyshev and Legendre polynomials exploiting Legendre-Galerkin, Legendre-collocation, Legendre-collocation penalty, 
Chebyshev-collocation, and Chebyshev-collocation penalty methods and compared the results among these methods. The differential Transform method is applied to compute eigenvalues and eigenfuctions of second order regular SLP's by Chen and Ho [2].The Weighted residual method using Chebyshev collocation points are investigated for approximate eigenvalues of second order SLP's by Ibrahim Celik [8]. Calculation of eigenvales of Helmholtz equation using boundary method are presented by Reutskiy [9]. The polynomial-based Differential Quadrature (PDQ) and the Fourier expansion-based differential quadrature (FDQ) methods are found in the work Ugur Yucel [10] to compute eigenvalues of the second order Sturm-Liouville problems. Chanane [6] used Shanon sampling theory to compute the eigenvalues of regular SLP's. The non linear Bratu problems are solved using various methods by different authors namely weighted residual [3], Domain Decomposition [4], B-Spline [13], Laplace transformation Decomposition [5], Decomposition [11], non-polynomial Spline [13], parametric Spline [15] and modified Adomian Decomposition [16] methods etc.

A class of singular SLP's are studied by Baily et al [1] applying the improved version of the algorithm of various proposed SLEIGN 2.

Application spectral methods in details for the solution of BVP's and SLP's are available in [7, 14, $18,19,20,21]$.

In this article we prefer Chebyshev Gauss-Lobatto points to compensate for Legendre GaussLobatto points .Since Legendre Gauss-Lobatto points are not explicitly defined and their estimation suffer round off errors for large $n$. Furthermore, discretizations with Chebyshev grid points with fairly fewer nodes reduce CPU time with a minimum effort. Since Chebyshev polynomials are mutually orthogonal with respect to a singular weight function $w(x)=\left(1-x^{2}\right)^{-1 / 2}$, which leads to complexities in the study of the Chebyshev spectral method. On the other hand, Legendre polynomials are mutually orthogonal in the standard $L^{2}$ inner product, with respect weight function $w(x)=1$, this criteria makes the Legendre spectral methods more attractive and much convenient for their analysis than that of the Chebyshev spectral method.

We organize this article as follows. In section 2, Chebyshev polynomials and Legendre polynomials together with their properties are introduced. In section 3 we discuss in brief about the spectral collocation methods. Section 4 is devoted for deriving the Spectral Legendre Operational Derivative matrix in precisely. Formulation of spectral collocation method and the techniques of imposing boundary conditions associated with SLP's are demonstrated in section 5. Convergence criteria are conferred in section 6. Section 7 includes some numerical results which verify of the accuracy of the current method. Finally conclusion is given.

\section{Legendre and Chebyshev polynomials}

The Legendre polynomials of degree $n$ defined on $[-1,1]$ is given as [17]:

$$
L_{n}(x)=\sum_{r=0}^{N}(-1)^{r} \frac{(2 n-2 r) !}{2^{n} r !(n-r) !(n-2 r) !} x^{n-2 r},
$$


where,

$N= \begin{cases}\frac{n}{2}, & \text { when } n \text { is even } \\ \frac{n-1}{2}, & \text { when } n \text { is odd }\end{cases}$

The Rodrigues' Formula of degree $n$ is defined as:

$L_{n}(x)=\frac{1}{2^{n} n !} \frac{d^{n}}{d x^{n}}\left(x^{2}-1\right)$, where $n \geq 1$

The $n$-th order Legendre differential equation is given by,

$-\left(\left(1-x^{2}\right) L_{n}^{\prime}(x)\right)^{\prime}=n(n+1) L_{n}(x)$

provided $L_{n}$ is bounded on [-1,1] i.e, $\left|L_{n}(x)\right| \leq 1$.

\section{Properties of Legendre polynomials}

(i) $L_{n}( \pm 1)=( \pm 1)^{n}$, (ii) $L_{n}^{\prime}( \pm 1)=\frac{1}{2}( \pm 1)^{n-1} n(n+1)$,

(iii) $\int_{-1}^{1} L_{n}(x) L_{r}(x) d x=\frac{\delta_{n r}}{n+\frac{1}{2}}, \forall r, n \geq 0$

The Legendre polynomials are orthogonal with respect to the $L^{2}(-1,1)$ inner product. Also these polynomials are complete in the sense that for any

$u(x)=\sum_{n=0}^{\infty} \tilde{u}_{n} L_{n}(x)$

$\tilde{u}_{n}=\left(n+\frac{1}{2}\right) \int_{-1}^{1} u(x) L_{n}(x) d x$

where, the sum converges to $L^{2}(-1,1)$ norm. Legendre polynomial which are orthogonal in the interval $[-1,1]$ satisfy the following recurrence relation.

$L_{n+1}(x)=\frac{2 n+1}{n+1} x L_{n}(x)-\frac{n}{n+1} L_{n-1}(x), n \geq 1$

Chebyshev polynomials [18] of degree $n$ over an interval $[a, b]$ is defined by

$$
T_{n}(x)=\cos \left(n \cos ^{-1}\left(\frac{2}{b-a}\left(x-\frac{a+b}{2}\right)\right)\right), n=0,1,2 \ldots \ldots \ldots . ., a \leq x \leq b .
$$

The collocation points $x_{k}$ in $[a, b]$ is defined as

$x_{k}=\frac{a-b}{2}\left(\frac{a+b}{b-a}+\cos \left(\frac{k \pi}{n}\right)\right), \quad k=0,1,2 \ldots \ldots \ldots, n$ 
$T_{n}(x)$ is bounded on $[-1,1]$. Chebyshev Gauss-Lobatto nodes are the zeroes of the orthogonal polynomial $\left(1-x^{2}\right) T_{n}^{\prime}(x)$. These nodes are placed symmetrically around $x=0$ and denser near the end points $x= \pm 1$.

\section{Legendre- Chebyshev collocation method}

The second order Sturm-Liouville eigenvalue problem is defined as:

$L u_{n}:=-\frac{d}{d x}\left(p(x) \frac{d u(x)}{d x}\right)+q(x) u(x)=\lambda \mu(x) u(x), \quad x \in(a, b)$

$p(x), q(x), \mu(x)>0$ are piecewise continuous functions and $L u_{n}$ is a self adjoint operator for the left hand side of equation(6a). Hence eigenvalues of a self-adjoint equation are all real.

Consider again the following homogeneous Sturm-Liouville boundary value problem (6a) specified as

$\frac{d}{d x}\left(x^{\gamma} \frac{d u}{\mathrm{~d} x}\right)=\lambda \mu(x) u(x), \quad a<x<b$

$u(a)=0$

$u(b)=0\}$

For every $\gamma>0$, the problem (6b) is called singular.

Let $x_{k}$ be the set of Gauss-Lobatto nodes with two end points $x_{0}$ and $x_{n}$, where $k=0,1,2, \ldots \ldots, n$ and let $p_{n}$ be the set of all real algebraic polynomials of degree $\leq n$. The spectral collocation method for equation (6a) is to find the $u_{n} \in p_{n}$ such that the residual $R_{n}(x)=L u_{n}(x)-\lambda \mu(x)$ equal to zero at the interior collocation points. The difference between its solution of any problem and its spectral approximations is of order $\frac{1}{n^{p}}$,where $p$ is the regularity index.

\section{Legendre Pseudospectral differentiation matrices :}

The present method is known as nodal method based on interpolation formulas that utilize Lagrange polynomials. Here unknowns are the actual sampled values of the function and so no transformation is needed.

$S_{L}(x)=\left(1-x^{2}\right) L_{n}^{\prime}(x)$

$S_{L}^{\prime}(x)=-2 x L_{n}^{\prime}(x)+\left(1-x^{2}\right) L_{n}^{\prime \prime}(x)$

Using equ.(3), we have

$S_{L}^{\prime}(x)=-n(n+1) L_{n}(x)$ 
Lagrange polynomial for the nodes $\left\{x_{0}, x_{1}, x_{2}, \ldots \ldots, x_{n}\right\}$ be defined as

$$
l_{i}(x)=\frac{\left(1-x^{2}\right) L_{n}^{\prime}(x)}{S_{L}^{\prime}\left(x_{i}\right)\left(x-x_{i}\right)}
$$

Since $\left\{x_{k}\right\}$ are the roots of $\left(1-x^{2}\right) L_{n}^{\prime}(x)$.

$$
\begin{aligned}
l_{i}\left(x_{i}\right) & =\frac{1}{S_{L}^{\prime}\left(x_{i}\right)} \operatorname{Lim}_{x \rightarrow x_{i}} \frac{\left(1-x^{2}\right) L_{n}^{\prime}(x)}{x-x_{i}}\left[\frac{0}{0} \text { form }\right] \\
& =\frac{1}{S_{L}^{\prime}\left(x_{i}\right)} \operatorname{Lim}_{x \rightarrow x_{i}} \frac{\left(1-x^{2}\right) L_{n}^{\prime \prime}(x)-2 x L_{n}^{\prime}(x)}{1} \quad \text { [using L' Hopital's rule] } \\
& =\frac{1}{S_{L}^{\prime}\left(x_{i}\right)} \operatorname{Lim}_{x \rightarrow x_{i}} S_{L}^{\prime}(x)=\frac{1}{S_{L}^{\prime}\left(x_{i}\right)} S_{L}^{\prime}\left(x_{i}\right)=1
\end{aligned}
$$

We can define

$$
\delta_{i k}= \begin{cases}0, & i \neq k \\ 1, & i=k\end{cases}
$$

Again differentiating equ.(10)

$$
\begin{aligned}
l_{i}^{\prime}(x) & =\frac{1}{S_{L}^{\prime}\left(x_{i}\right)} \frac{\left(x-x_{i}\right)\left\{L_{n}^{\prime \prime}(x)-2 x L_{n}^{\prime}(x)\right\}-\left(1-x^{2}\right) L_{n}^{\prime}(x)}{\left(x-x_{i}\right)^{2}} \\
& =\frac{1}{S_{L}^{\prime}\left(x_{i}\right)} \frac{\left(x-x_{i}\right) S_{L}^{\prime}(x)-S_{L}(x)}{\left(x-x_{i}\right)^{2}} \\
l_{i}^{\prime}(x) & =\frac{1}{S_{L}^{\prime}\left(x_{i}\right)}\left[\frac{S_{L}^{\prime}(x)}{x-x_{i}}-\frac{S_{L}(x)}{\left(x-x_{i}\right)^{2}}\right]
\end{aligned}
$$

For any continuous function $u$, we define Legendre interpolate of $u$ by $I_{n}^{L} u$, can be expressed as the unique polynomial in $p_{n}$ such that,

$$
\left(I_{n}^{L} u\right)(x)=\sum_{i=0}^{n} u_{i} l_{i}(x), \quad 0 \leq k \leq n \quad \text { and } \quad\left(I_{n}^{L} u\right)^{\prime}(x)=\sum_{i=0}^{n} u_{i} l_{i}^{\prime}(x)
$$

From equ.(13)

$$
\begin{aligned}
l_{i}^{\prime}\left(x_{k}\right)=\frac{1}{S_{L}^{\prime}\left(x_{i}\right)} \frac{S_{L}^{\prime}\left(x_{k}\right)}{\left(x_{k}-x_{i}\right)} & =\frac{1}{-n(n+1) L_{n}\left(x_{i}\right)} \frac{-n(n+1) L_{n}\left(x_{k}\right)}{L_{n}\left(x_{k}-x_{i}\right)}, \quad \text { since } S_{L}\left(x_{k}\right)=0 \\
& =\frac{L_{n}\left(x_{k}\right)}{L_{n}\left(x_{i}\right)\left(x_{k}-x_{i}\right)}, \quad i \neq k
\end{aligned}
$$

Also, $D_{i, i}=l_{i}^{\prime}\left(x_{i}\right)=\frac{1}{S_{L}^{\prime}\left(x_{i}\right)} \lim _{x \rightarrow x_{i}} \frac{S_{L}^{\prime}(x)\left(x-x_{i}\right)-S_{L}(x)}{\left(x-x_{i}\right)^{2}}=\frac{S_{L}^{\prime \prime}\left(x_{i}\right)}{2 S_{L}^{\prime}\left(x_{i}\right)}=\frac{L_{n}^{\prime}\left(x_{k}\right)}{2 L_{n}\left(x_{k}\right)}$

Equation (16) is obtained using equ. (9) and L' Hospital Rule. 
$D_{k, k}=0$ for $1 \leq k \leq n-1, \quad D_{0,0}=-D_{n, n}=\frac{n(n+1)}{4}$.

Equation (17) is obtained using properties of Legendre polynomials illustrated in section 2. It can be shown that the $(n+1) \times(n+1)$ Legendre Pseudospectral derivative matrix $D$, which computes the derivative exactly at the Legendre Gauss-Lobatto nodes, gives the derivative of the interpolate of $u$. Using Equ.(15),(16) and (17) ,the Legendre Pseudospectral derivative can be written together as,

$D_{k, i}=\left\{\begin{array}{l}\frac{1}{x_{k}-x_{i}} \frac{L_{n}\left(x_{k}\right)}{L_{n}\left(x_{i}\right)} \quad, i \neq k \\ 0, \quad 1 \leq i=k \leq n-1 \\ \frac{n(n+1)}{4}, \quad i=k=0 \\ -\frac{n(n+1)}{4}, i=k=n ;\end{array}\right.$

\section{Formulation of second order SLP's}

Equation (9a) are to be put in the form as follows:

$u^{\prime \prime}\left(x_{k}\right)+p\left(x_{k}\right) u^{\prime}\left(x_{k}\right)+q\left(x_{k}\right) u-\lambda \mu\left(x_{k}\right) u=0, \quad 1 \leq k \leq n-1$

General boundary conditions (mixed type) are written as:

$$
\begin{aligned}
\alpha_{1} u_{n}+\beta_{1} \sum_{k=0}^{n}\left(D^{1}\right)_{n k} u_{k} & =0 \\
\alpha_{2} u_{0}+\beta_{2} \sum_{k=0}^{n}\left(D^{1}\right)_{0 k} u_{k} & =0
\end{aligned}
$$

Equation (20a) leads to

$\beta_{1}\left(D^{1}\right)_{n 0} u_{0}+\left[\alpha_{1}+\beta_{1}\left(D^{1}\right)_{n n}\right] u_{n}=-\beta_{1} \sum_{k=1}^{n-1}\left(D^{1}\right)_{n k} u_{k}$

Similarly from (20b), we have

$$
\begin{aligned}
& {\left[\left(\alpha_{2}+\beta_{2}\left(D^{1}\right)_{00}\right] u_{0}+\beta_{2}\left(D^{1}\right)_{0 n} u_{n}=-\beta_{2} \sum_{k=1}^{n-1}\left(D^{1}\right)_{0 k} u_{k}, \quad a \leq x \leq b\right.} \\
& u_{0}=\frac{-\beta_{2} \sum_{k=1}^{n-1}\left(D^{1}\right)_{0 k} u_{k}-\beta_{2}\left(D^{1}\right)_{0 n} u_{n}}{\alpha_{2}+\beta_{2}\left(D^{1}\right)_{00}}
\end{aligned}
$$

Substituting equation (22) into equation (21a),

$$
\beta_{1}\left(D^{1}\right)_{n 0} \times\left[\frac{-\beta_{2}\left(D^{1}\right)_{0 n} u_{n}}{\left(\alpha_{2}+\beta_{2}\left(D^{1}\right)_{00}\right.}-\frac{\beta_{2} \sum_{k=1}^{n-1}\left(D^{1}\right)_{0 k} u_{k}}{\left(\alpha_{2}+\beta_{2}\left(D^{1}\right)_{00}\right.}\right]+\left[\alpha_{1}+\beta_{1}\left(D^{1}\right)_{n n}\right] u_{n}=-\beta_{1} \sum_{k=1}^{n-1}\left(D^{1}\right)_{n k} u_{k}
$$


Now let,

$$
a_{1}=\beta_{1}\left(D^{1}\right)_{n 0}, b_{1}=\alpha_{1}+\beta_{1}\left(D^{1}\right)_{n n}, c_{1}=\alpha_{2}+\beta_{2}\left(D^{1}\right)_{00}, d_{1}=\beta_{2}\left(D^{1}\right)_{0 n}
$$

Using equation (24), equation (23) becomes

$$
\begin{aligned}
& a_{1} \times\left[\frac{-d_{1} u_{n}}{c_{1}}-\frac{\beta_{2} \sum_{k=1}^{n-1}\left(D^{1}\right)_{0 k} u_{k}}{c_{1}}\right]+b_{1} u_{n}=-\beta_{1} \sum_{k=1}^{n-1}\left(D^{1}\right)_{n k} u_{k} \\
& \frac{\left(b_{1} c_{1}-a_{1} d_{1}\right)}{c_{1}} u_{n}=\frac{-\beta_{1} c_{1} \sum_{k=1}^{n-1}\left(D^{1}\right)_{n k} u_{k}}{c_{1}}+\frac{a_{1} \beta_{2} \sum_{k=1}^{n-1}\left(D^{1}\right)_{0 k} u_{k}}{c_{1}}
\end{aligned}
$$

$u_{n}=\frac{\beta_{1} c_{1} \sum_{k=1}^{n-1}\left(D^{1}\right)_{n k} u_{k}}{a_{1} d_{1}-b_{1} c_{1}}-\frac{a_{1} \beta_{2} \sum_{k=1}^{n-1}\left(D^{1}\right)_{0 k} u_{k}}{a_{1} d_{1}-b_{1} c_{1}}$

$u_{n}=\sum_{k=1}^{n-1} \theta_{n k} u_{k}$

where,

$\theta_{n k}=\frac{c_{1} \beta_{1}\left(D^{1}\right)_{n k}-a_{1} \beta_{2}\left(D^{1}\right)_{0 k}}{a_{1} d_{1}-b_{1} c_{1}}$

Also from equation (22)

$$
u_{0}=-\frac{d_{1}}{c_{1}} u_{n}-\frac{\beta_{2} \sum_{k=1}^{n-1}\left(D^{1}\right)_{0 k} u_{k}}{c_{1}}=-\frac{d_{1}}{c_{1}}\left\{\frac{\beta_{1} c_{1} \sum_{k=1}^{n-1}\left(D^{1}\right)_{n k} u_{k}-a_{1} \beta_{2} \sum_{k=2}^{n-1}\left(D^{1}\right)_{0 k} u_{k}}{a_{1} d_{1}-b_{1} c_{1}}\right\}-\frac{\beta_{2} \sum_{k=1}^{n-1}\left(D^{1}\right)_{0 k} u_{k}}{c_{1}}
$$

On simplification

$$
\begin{aligned}
& u_{0}=-\frac{\left(\beta_{1} d_{1} \sum_{k=1}^{n-1}\left(D^{1}\right)_{n k}-b_{1} \beta_{2} \sum_{k=1}^{n-1}\left(D^{1}\right)_{0 k}\right) u_{k}}{\left(a_{1} d_{1}-b_{1} c_{1}\right)} \\
& u_{0}=\sum_{k=1}^{n-1} \theta_{0 k} u_{k}
\end{aligned}
$$

where $\theta_{0 k}=\frac{b_{1} \beta_{2}\left(D^{1}\right)_{0 k}-\beta_{1} d_{1}\left(D^{1}\right)_{n k}}{\left(a_{1} d_{1}-b_{1} c_{1}\right)}$

Let the constants $\beta_{1}$ and $\beta_{2}$ be nonzero.

Using (25b) and (26b) the equation (19) reduces to 


$$
\begin{aligned}
& \sum_{k=1}^{n-1}\left[\left(D^{2}\right)_{i, k}+p\left(x_{i}\right)\left(D^{1}\right)_{i, k}+\left\{q\left(x_{i}\right)-\lambda \mu\left(x_{i}\right) u\left(x_{i}\right)\right\} \delta_{i, k}\right] u_{k} \\
& +\left[\left(D^{2}\right)_{i, 0}+p\left(x_{i}\right)\left(D^{1}\right)_{i, 0}+q\left(x_{i}\right)-\lambda \mu\left(x_{i}\right)\right]\left[\sum_{k=1}^{n-1} \theta_{0 k} u_{k}\right]+ \\
& +\left[\left(D^{2}\right)_{i, n}+p\left(x_{i}\right)\left(D^{1}\right)_{i, n}+q\left(x_{i}\right)-\lambda \mu\left(x_{i}\right)\right]\left[\sum_{k=1}^{n-1} \theta_{n k} u_{k}\right]=0, \\
& =\sum_{k=1}^{n-1}\left[\left(D^{2}\right)_{i, k}+p\left(x_{i}\right)\left(D^{1}\right)_{i, k}+\left\{q\left(x_{i}\right)-\lambda \mu\left(x_{i}\right) u\left(x_{i}\right)\right\} \delta_{i, k}\right] u_{k} \\
& \quad-\left[\left(D^{2}\right)_{i 0}+p\left(x_{i}\right)\left(D^{1}\right)_{i 0}+q\left(x_{i}\right)\right] \theta_{0 k} u_{k}-\left[\left(D^{2}\right)_{i n}+p\left(x_{i}\right)\left(D^{1}\right)_{i n}+q\left(x_{i}\right)\right] \theta_{n k} u_{k} \\
& +\lambda \mu\left(x_{i}\right)\left(\theta_{0 k}+\theta_{n k}\right) u_{k}=0
\end{aligned}
$$

The spectral collocation solution for the eigenvalues for the SLP (19) with the general boundary conditions (20a) and (20b) takes the matrix equation form as given by

$$
A \tilde{u}=\lambda B \tilde{u}
$$

where,

$$
\begin{aligned}
a_{i, k}= & \left(D^{2}\right)_{i, k}+p\left(x_{i}\right)\left(D^{1}\right)_{i, k}+\left\{q\left(x_{i}\right)-\lambda \mu\left(x_{i}\right) u\left(x_{i}\right)\right\} \delta_{i, k}-\left[\left(D^{2}\right)_{i 0}+p\left(x_{i}\right)\left(D^{1}\right)_{i 0}+q\left(x_{i}\right)\right] \theta_{0 k} \\
& -\left[\left(D^{2}\right)_{i n}+p\left(x_{i}\right)\left(D^{1}\right)_{i n}+q\left(x_{i}\right)\right] \theta_{n k} \\
b_{i, k}= & \mu\left(x_{i}\right)\left(\theta_{0 k}+\theta_{n k}\right)
\end{aligned}
$$

Here,

$\tilde{u}=\left[u_{1}, u_{2}, \ldots \ldots \ldots u_{n-1}\right]^{T}, \quad A=\left(a_{i . k}\right), \quad B=\left(b_{i, k}\right) \quad$ are $(n-1) \times(n-1)$ matrices, and $\delta_{i, k}$ is the Kronecker delta. Solving eqnuation (28) the required eigenvalues are obtained.

\section{SLP's with Dirichlet boundary conditions:}

The Dirichlet boundary conditions are:

$u(a)=0 ; \quad u(b)=0$

The spectral differentiation matrix for the SLP (19) incorporating the boundary conditions (29)

$\sum_{k=1}^{n-1}\left[\left(D^{2}\right)_{i, k}+p\left(x_{i}\right)\left(D^{1}\right)_{i, k}+\left\{q\left(x_{i}\right)-\lambda \mu\left(x_{i}\right)\right\} \delta_{i, k}\right] u_{k}=0$

Dirichlet boundary conditions (29) satisfies the following linear system

$$
A \tilde{u}=\lambda B \tilde{u}
$$

where,

$$
\begin{aligned}
& \tilde{u}=\left[u_{1}, u_{2,}, \ldots \ldots . . u_{n-1}\right]^{T} \\
& a_{i, k}=\left(D^{2}\right)_{i, k}+p\left(x_{i}\right)\left(D^{1}\right)_{i, k}+q\left(x_{i}\right) \delta_{i, k}
\end{aligned}
$$


$b_{i, k}=\mu\left(x_{i}\right) \delta_{i, k}$

Solving equ. (31) required eigenvalues are obtained.

\section{Convergence analysis}

The Legendre polynomials, $L_{k}(x), \quad k=0,1,2 \ldots \ldots \ldots \ldots$ form eigenfunctions of singular SturmLiouville problems given as

$\left(\left(1-x^{2}\right) L_{k}^{\prime}(x)\right)^{\prime}+k(k+1) L_{k}(x)=0$.

If $\left\{L_{k}(x)\right\}$ be a set of orthogonal polynomials with respect to weight function $w(x)$, then

$\int_{-1}^{1} L_{k}(x) L_{l}(x) w(x) d x=0, \quad$ for $k \neq l$

We consider $\phi(x)$ be the functions in the Hilbert space $L_{w}^{2}(-1,1)$ such that

$$
\int_{-1}^{1}|\phi(x)|^{2} w(x) d x<\infty
$$

For any continuous functions $\phi(x)$ and $\psi(x)$ in $L_{w}^{2}(-1,1)$, we have

$$
\langle\phi, \psi\rangle=\int_{-1}^{1} \phi(x) \psi(x) w(x) d x
$$

Suppose, $u(x)$ be the eigenfunction of the Sturm-Liouville problem in the Hilbert space then the series expansion in the case of Legendre polynomials is

$u(x)=\sum_{k=0}^{\infty} \tilde{u}_{k} L_{k}(x)$

Approximate solution in terms of truncated Legendre series is

$u(x)=\sum_{k=0}^{n} \tilde{u}_{k} L_{k}(x)$

where the coefficients, $\tilde{u}_{k}=\frac{\int_{-1}^{1} u(x) L_{k}(x) w(x) d x}{\left\|L_{k}(x)\right\|^{2}}$,

since $w(x)=1$, for Legendre polynomials, we have,

$$
\left\|L_{k}\right\|_{L_{w}^{2}(-1,1)}^{2}=\int_{-1}^{1}\left|L_{k}\right|^{2} d x
$$

If $P_{n}$ be the orthogonal projection operator onto the Legendre polynomial space $\Pi_{n}$, then

$$
\left(P_{n} \tilde{u}, v\right)_{w}=(u, v)_{w}, \forall v \in \Pi_{n}
$$

The completeness of $\left\{L_{k}(x)\right\}$ implies that, 


$$
\left\|u-P_{n} u\right\| \rightarrow 0 \text { as } n \rightarrow \infty . \quad \forall u \in L_{w}^{2}(-1,1) .
$$

Hence, following the above convergence result, if $\forall u \in C^{\infty}$, the produced error approaches to zero as $n \rightarrow \infty$ and with exponential rate $O\left(\lambda^{m} e^{-\gamma n}\right), \quad \gamma>0$, for the $m$-th eigenvalue [18,19].

\section{Numerical Experiments}

In this section we present five numerical examples of second order Sturm-Liouville problems, using the method, outlined in the previous section. The convergence of the our existing method is measured by the absolute error

$$
\delta_{k}=\left|\lambda^{\text {exact }}-\lambda^{(\text {coll. })}\right| \text { and the relative error }: \varepsilon_{k}=\left|\frac{\lambda^{\text {exact }}-\lambda^{(\text {coll } .)}}{\lambda^{\text {exact }}}\right| \text {. }
$$

Example 1. Let us consider one dimensional Helmholtz equation [9]

$$
\left.\begin{array}{l}
\frac{d^{2} y}{d x^{2}}=\lambda^{2} y \\
y(0)=y(1)=0
\end{array}\right\}
$$

We transfer the equation (43a) by changing the variables $x=\frac{1}{2} t+\frac{1}{2}$, the Sturm-Liouville problem transforms to

$$
\left.\begin{array}{l}
4 \frac{d^{2} y}{d t^{2}}=\lambda y \quad, t \in(-1,1) \\
y(-1)=y(1)=0
\end{array}\right\}
$$

Table 1: Comparison of absolute errors between the new boundary method ( $\varepsilon$-procedure) [9] and present method for example 1

\begin{tabular}{|c|c|c|c|c|}
\hline$x$ & $\begin{array}{c}\text { Exact } \\
\text { eigenvalues }\end{array}$ & $\begin{array}{c}\text { Relative errors of the present } \\
\text { method for } n=20\end{array}$ & $\begin{array}{c}\text { Relative errors of the } \\
\text { present method for } n=30\end{array}$ & $\begin{array}{c}\text { Relative errors } \\
{[9], \varepsilon=10^{-6}}\end{array}$ \\
\hline 1 & $\pi$ & $3.251 \mathrm{e}-14$ & $3.251 \mathrm{e}-014$ & $1.7 \mathrm{e}-012$ \\
\hline 2 & $2 \pi$ & $3.209 \mathrm{e}-13$ & $3.223 \mathrm{e}-013$ & $1.6 \mathrm{e}-012$ \\
\hline 3 & $3 \pi$ & $2.344 \mathrm{e}-13$ & $2.344 \mathrm{e}-013$ & $1.5 \mathrm{e}-012$ \\
\hline 4 & $4 \pi$ & $3.906 \mathrm{e}-14$ & $8.371 \mathrm{e}-015$ & $9.7 \mathrm{e}-013$ \\
\hline 5 & $5 \pi$ & $2.790 \mathrm{e}-13$ & $7.924 \mathrm{e}-014$ & $9.0 \mathrm{e}-013$ \\
\hline 6 & $6 \pi$ & $2.353 \mathrm{e}-10$ & $4.278 \mathrm{e}-014$ & $5.8 \mathrm{e}-013$ \\
\hline 7 & $7 \pi$ & $5.236 \mathrm{e}-08$ & $1.594 \mathrm{e}-014$ & $9.2 \mathrm{e}-013$ \\
\hline 8 & $8 \pi$ & $3.706 \mathrm{e}-06$ & $8.371 \mathrm{e}-015$ & $1.8 \mathrm{e}-013$ \\
\hline 9 & $9 \pi$ & $1.450 \mathrm{e}-04$ & $1.240 \mathrm{e}-014$ & $5.3 \mathrm{e}-013$ \\
\hline 10 & $10 \pi$ & $2.168 \mathrm{e}-03$ & $1.671 \mathrm{e}-012$ & $1.2 \mathrm{e}-012$ \\
\hline
\end{tabular}


The Differential eigenvalue problems, in matrix form, can be written together with boundary conditions as

$\sum_{k=1}^{n-1}\left[\left(D^{2}\right)_{i, k}-\lambda \delta_{i, k}\right] y_{k}=0$

Table1, lists first ten eigenvalues for $n=20$. Smallest eigenvalue attains the accuracy upto $10^{-14}$ and error increases rapidly for higher eigenvalues than the lower values which is better than boundary method. As we increase the grid points or nodes from $n=20$ to $n=30$, the error decreases very fast for all the eigenvalues and accuracy is obtained upto $10^{-15}$. We observe that increasing of nodes reveal the stable behaviour of all the eigenvalues for $n=30$.

From table 1, it is observed that our present approach attains more accurate results than the new boundary approach for one dimensional Helmholtz equation.

Example 2. Consider the SLP studied by Celik [8] as below

$$
\left.\begin{array}{l}
\frac{d^{2} y}{d x^{2}}+\left(\lambda-e^{x}\right) y=0 \\
y(0)=y(\pi)=0
\end{array}\right\}
$$

changing the variables $x=\frac{\pi}{2} t+\frac{1}{2}$, the Sturm-Lioville problem (45a) transforms to

$$
\left.\begin{array}{l}
\frac{4}{\pi^{2}} \frac{d^{2} y}{d x^{2}}-y e^{\left.\frac{\pi}{2}(t+1)\right)}=\lambda y, t \in(-1,1) \\
y(-1)=y(1)=0
\end{array}\right\}
$$

The Differential eigenvalue problems in matrix form can be written together with boundary conditions as

$$
\sum_{k=1}^{n-1}\left[\left(D^{2}\right)_{i, k}-e^{\frac{\pi}{2}\left(t_{k}+1\right)}\right] y_{k}=\lambda \delta_{i, k} y_{k}
$$

Absolute errors obtained by using PDQ, FDQ, Chebyshev collocation and our present method are depicted in Table 2 for $n=40$. Yucel [10] showed that FDQ approach gives better convergence than that of DQ. We achieve almost the same accuracy in our existing method is. Thus our present approach is in good agreement with the other three other methods and much accurate.

Example 3. This SLP is taken from the article worked out by Chen and Ho [2]

$$
\begin{aligned}
& \frac{d^{2} y}{d x^{2}}=\lambda y \\
& y(0)-y^{\prime}(0)=0 \\
& y(1)+y^{\prime}(1)=0
\end{aligned}
$$


Table 2. Comparison of absolute errors between the Chebyshev's collocation and present method for example 2

\begin{tabular}{c|c|c|c|c|c|c|c}
\hline$k$ & $\begin{array}{c}\text { Exact } \\
\text { eigenvalues }\end{array}$ & $\begin{array}{c}\text { Chebyshev } \\
\text { coll. [8] } \\
N=40\end{array}$ & $\begin{array}{c}\text { Spectral } \\
\text { collocation } \\
\text { present } \\
N=40\end{array}$ & $\begin{array}{c}\text { Absolute error } \\
\text { Chebyshev } \\
\text { Coll. [8] } \\
N=40\end{array}$ & $\begin{array}{c}\text { Absolute } \\
\text { error } \\
\text { PDQ [10] } \\
N=40\end{array}$ & $\begin{array}{c}\text { Absolute } \\
\text { error } \\
\text { FDQ [10] } \\
N=40\end{array}$ & $\begin{array}{c}\text { Absolute } \\
\text { error Spect. } \\
\text { Collocation } \\
\text { (present) }\end{array}$ \\
\hline 1 & 4.8966694 & 4.8966694 & 4.896694 & 0.00000 & 0.0000 & 0.0000 & 0.000000 \\
\hline 2 & 10.045190 & 0.045190 & 10.045190 & 0.00000 & 0.0000 & 0.0000 & 0.000000 \\
\hline 3 & 16.019267 & 6.019267 & 16.019267 & 0.00000 & 0.0000 & 0.0000 & 0.000000 \\
\hline 4 & 23.266271 & 3.266271 & 23.266271 & 0.00000 & 0.0000 & 0.0000 & 0.000000 \\
\hline 5 & 32.263707 & 2.263707 & 32.263707 & 0.00000 & 0.0000 & 0.0000 & 0.000000 \\
\hline 6 & 43.220020 & 3.220020 & 88.132119 & 0.00000 & 0.0000 & 0.0000 & 0.000000 \\
\hline 7 & 56.181594 & 6.181594 & 56.181594 & 0.00000 & 0.0000 & 0.0000 & 0.000000 \\
\hline 8 & 71.152998 & 1.152998 & 71.152998 & 0.00000 & 0.0000 & 0.0000 & 0.000000 \\
\hline 9 & 88.132119 & 8.132119 & 88.132119 & 0.00000 & 0.0000 & 0.0000 & 0.000000 \\
\hline 10 & 107.11668 & 07.11668 & 107.11668 & 0.00000 & 0.0000 & 0.0000 & 0.000000 \\
\hline 11 & 128.10502 & 128.10502 & 128.10502 & 0.00000 & 0.0000 & 0.0000 & 0.000000 \\
\hline 12 & 151.09604 & 51.09604 & 151.09604 & 0.00000 & 0.0000 & 0.0000 & 0.000000 \\
\hline 13 & 176.08900 & 76.08900 & 176.08900 & 0.00000 & 0.0000 & 0.0000 & 0.000000 \\
\hline 14 & 203.08337 & 03.08337 & 203.08337 & 0.00000 & 0.0000 & 0.0000 & 0.000000 \\
\hline 15 & 232.07881 & 32.07881 & 232.07881 & 0.00000 & 0.000 & 0.0000 & 0.000000 \\
\hline 16 & 263.07507 & 63.07507 & 263.07507 & 0.00000 & 0.010 & 0.0000 & 0.00000 \\
\hline 17 & 296.07196 & 296.07198 & 296.07196 & 0.00002 & 0.020 & 0.0000 & 0.00000 \\
\hline 18 & 331.06934 & 31.06940 & 331.06935 & 0.00005 & 0.0500 & 0.0000 & 0.00001 \\
\hline 19 & 368.06713 & 68.06769 & 368.06702 & 0.00052 & 0.5600 & 0.0000 & 0.000011 \\
\hline 20 & 407.06524 & 07.04923 & 407.06672 & 0.01502 & 16.01 & 0.030 & 0.00148 \\
\hline & & & & & & & \\
\hline
\end{tabular}

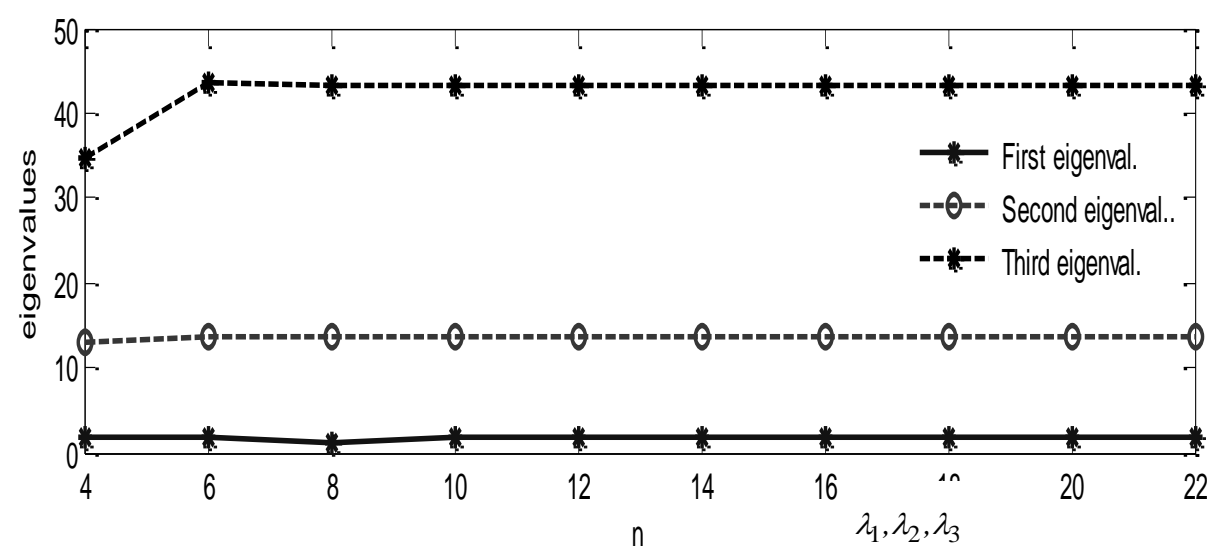

Figure 1: Convergence of eigenvalues 
Change the boundary points from 0 to 1 into -1 to 1 , leads the SLP as follows:

$$
\begin{aligned}
& 4 \frac{d^{2} y}{d x^{2}}=\lambda y \\
& y(-1)-y^{\prime}(-1)=0 \\
& y(1)+y^{\prime}(1)=0
\end{aligned}
$$

Here $\lambda_{k}^{n}$ is k-th estimated eigenvalue corresponding to $n$ and the differences between the $k$-th and

\begin{tabular}{|c|c|c|c|}
\hline$i$ & $\begin{array}{c}\text { Exact eigevalues } \\
\text { Chen and Ho } \\
{[2]}\end{array}$ & $\begin{array}{c}\text { Absolute } \\
\text { error present } \\
\left|\lambda_{i}^{6}-\lambda_{i}^{5}\right|\end{array}$ & $\begin{array}{c}\text { Absolute } \\
\text { error present } \\
\left|\lambda_{i}^{12}-\lambda_{i}^{11}\right|\end{array}$ \\
\hline 1 & 1.71 & $2.96 \mathrm{e}-004$ & $1.50 \mathrm{e}-012$ \\
\hline 2 & 13.49 & $5.19 \mathrm{e}-002$ & $5.97 e-008$ \\
\hline 3 & 43.36 & $5.29 \mathrm{e}-001$ & $7.16 \mathrm{e}-004$ \\
\hline
\end{tabular}
(k-1)-th eigenvalues are given by $\left|\lambda_{i}^{k}-\lambda_{i}^{k-1}\right|<\varepsilon$, where $\varepsilon$ is very small and $\varepsilon \rightarrow 0$.

Table 3. Absolute errors between the successive eigenvalues for example 3

It is observed from Table 3 that the differences between successive eigenvalues converge to zero as the node number inceased and is given as follows:

$$
\left|\lambda_{1}^{12}-\lambda_{1}^{11}\right| \leq 0.000001, \quad\left|\lambda_{2}^{12}-\lambda_{2}^{11}\right| \leq 0.000001 \text { and }\left|\lambda_{3}^{12}-\lambda_{3}^{11}\right| \leq 0.000001
$$

Chen and Ho [2] computed absolute differences between the successive eigenvalues and found that these differences tend to zero as he increased the order of derivatives. We also calculate the absolute differences and relative errors of the first three eigenvalues for $n=5,6$ and $n=11,12$. It is clear that absolute differences diminish by zero as the node numbers are increased.

Example 4. Consider the singular Sturm-Liouville boundary value problem illustrated in the article of Singh and Kumar [16]

$$
\left.\begin{array}{l}
-\frac{d^{2} y}{d x^{2}}=\lambda^{2} \frac{1}{x} y(x) \quad, \quad 0<x \leq 1 \\
y(0)=y(1)=0
\end{array}\right\}
$$

The exact eigenvalues are computed solving the equation of the Bessel function given as. We compare nine approximate eigenvalues for $n=20$ nodes with those tabulated using Adomian Decomposition method [16]. 
Table 4: Comparison of eigenvalues obtained by present method and ADM for example 4.

\begin{tabular}{c|c|c|c}
\hline$k$ & Exact eigenvalues $\lambda_{k}$ & Present (spectral Coll.) $\lambda_{k}$ & Spectral Coll. ADM [16] $\lambda_{k}$ \\
\hline 1 & 1.9158529 & 1.9158529851 & 1.9158529 \\
\hline 2 & 3.5077933 & 3.5077933349 & 3.5077933 \\
\hline 3 & 5.0867340 & 5.0867340674 & 5.0867340 \\
\hline 4 & 6.6618459 & 6.6618459681 & 6.6618459 \\
\hline 5 & 8.2353150 & 8.2353150254 & 8.2353150 \\
\hline 6 & $\ldots \ldots \ldots \ldots$ & 9.8079292552 & 9.8079292 \\
\hline 7 & $\ldots \ldots \ldots \ldots$ & 11.3800421902 & 11.380042 \\
\hline 8 & $\ldots \ldots \ldots \ldots$ & 12.9518360438 & 12.9518360 \\
\hline $\mathbf{9}$ & $\ldots \ldots \ldots \ldots$ & 14.5235414260 & 14.5235964 \\
\hline
\end{tabular}

From Table 4, it is noticed that the first eight numerically attained eigenvalues by our present method are correct upto figures eight significant which is yields reasonable accuracy.

Example 5: The Boyd equation considered by Baily et al [1], Singh and Kumar [16]

$$
\left.\begin{array}{l}
-\frac{d^{2} y}{d x^{2}}=\lambda y(x)+\frac{1}{x} y(x) \\
y(0)=y(1)=0
\end{array}\right\}
$$

Table 5: Comparison of solutions obtained by present method for Boyd equation with other methods for example 5.

\begin{tabular}{c|c|c}
\hline$k$ & $\begin{array}{c}\text { Our method } \\
n=20\end{array}$ & $\begin{array}{c}\text { Adomian } \\
\text { Decomposition }\end{array}$ \\
\hline 1 & 7.3739850 & 7.3739850 \\
\hline 2 & 36.3360196 & 36.3360196 \\
\hline 3 & 85.2925821 & 85.2925820 \\
\hline 4 & 154.0986237 & 154.0986237 \\
\hline 5 & 242.7055594 & 242.7055594 \\
\hline
\end{tabular}

From Table 5, we observe that eigenvalues work out by our present approach agrees well with the Adomian Decomposition method.

Example 6. Consider one dimensional Bratu nonlinear problem studied by some authors $[3,5,11$, $12,13,15,21]$ for $\lambda>0$ :

$$
\left.\begin{array}{l}
\frac{d^{2} y}{d x^{2}}=\lambda e^{y(x)} \\
y(0)=y(1)=0
\end{array}\right\}
$$

The exact solutions of (50a) is found as $y(x)=-2 \ln \left[\cosh \left\{\frac{\theta}{2}\left(x-\frac{1}{2}\right)\right\} / \cosh \left(\frac{\theta}{4}\right)\right]$ 
Solving the equation $\theta=\sqrt{2 \lambda} \operatorname{coh}(\theta / 4)$, the values $\theta$ are computed.

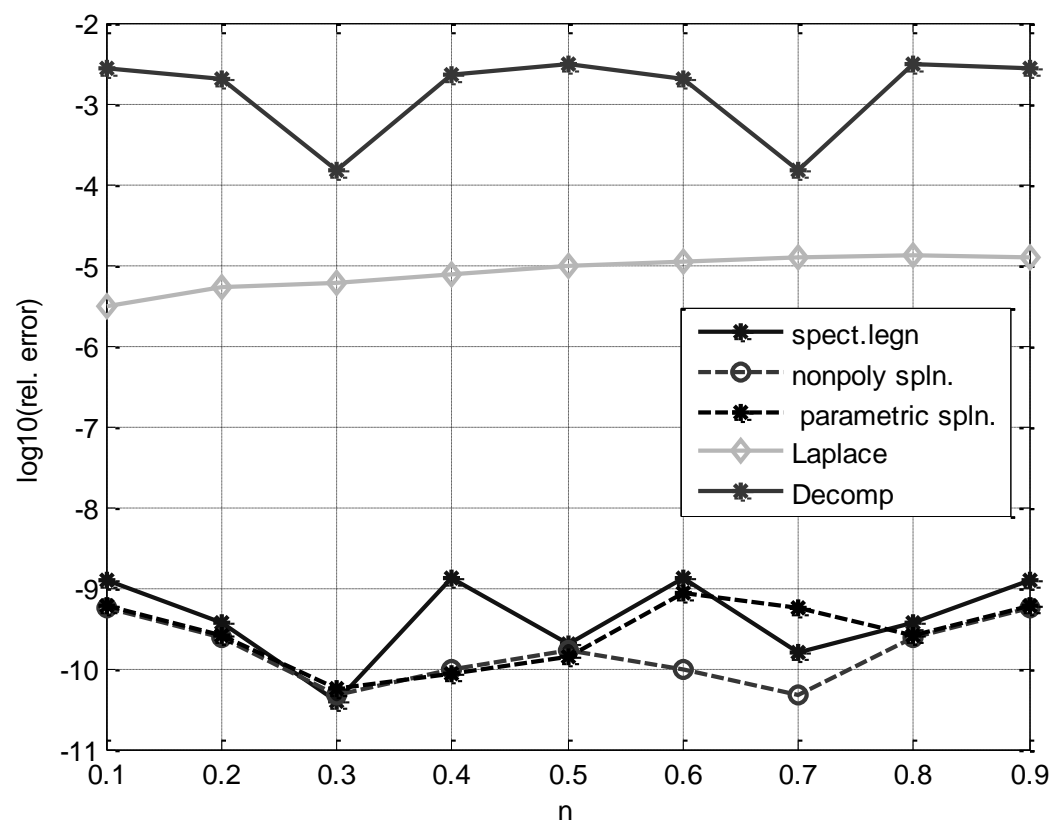

Fig. 2: Comparison of our results obtained using spectral collocation method with the nonpolyspline, parametric spline and Laplace transformation decomposition methods for $n=10$ and $\lambda=1$.

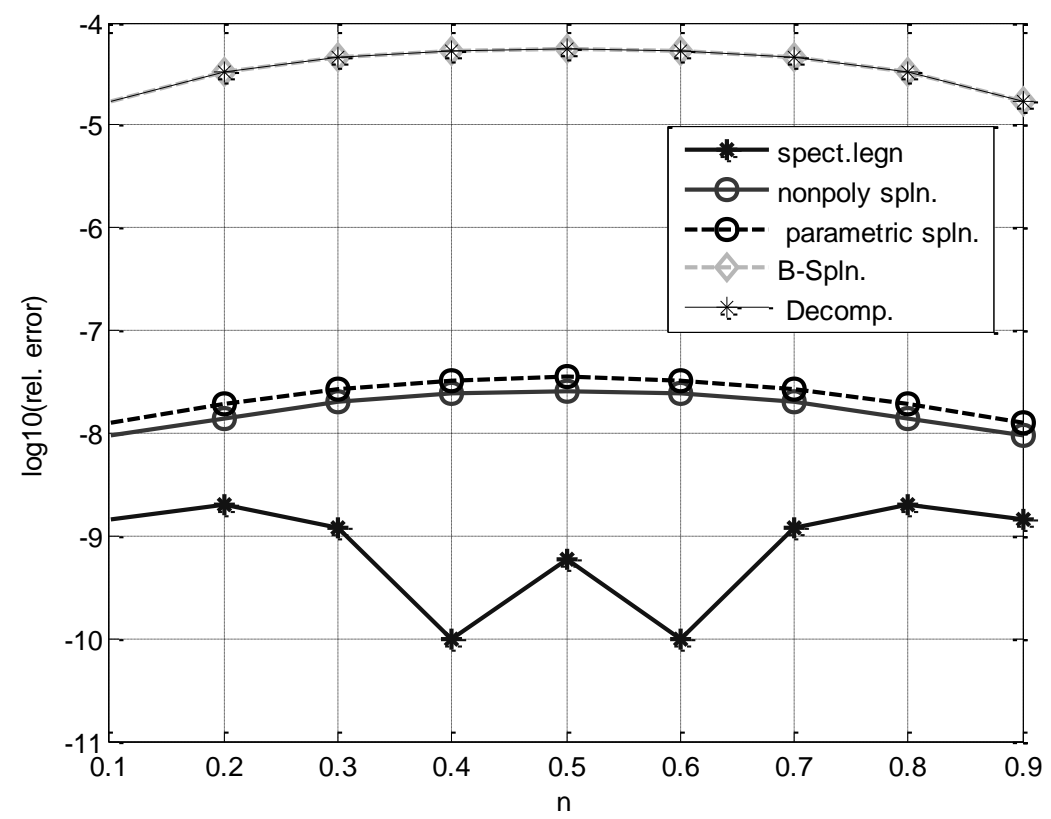

Fig. 3: Comparison of our result obtained using spectral collocation method with the nonpoly-spline, parametric spline and B-spline methods for $n=10$ and $\lambda=2$. 
Table 6: Comparison of solutions obtained by present method for Bratu equation with other methods for $\lambda=$ 1 for example 6 .

\begin{tabular}{c|l|l|l|l|l|l|l}
\hline$x$ & $\begin{array}{c}\text { Exact } \\
\text { eigenvalues }\end{array}$ & $\begin{array}{c}\text { Present } \\
\text { method } \\
N=10\end{array}$ & $\begin{array}{c}\text { Parametric } \\
\text { spline [15] } \\
N=10\end{array}$ & $\begin{array}{c}\text { Non polyn. } \\
\text { Spline [13] } \\
N=10\end{array}$ & $\begin{array}{c}\text { Laplace } \\
{[5]}\end{array}$ & $\begin{array}{c}\text { Decomp. } \\
{[11]}\end{array}$ & $\begin{array}{c}\text { B-spline } \\
{[12]}\end{array}$ \\
\hline 0.1 & 0.0498467900 & $1.24 \mathrm{e}-009$ & $5.87 \mathrm{e}-010$ & $5.77 \mathrm{e}-010$ & $1.98 \mathrm{e}-06$ & $2.68 \mathrm{e}-03$ & $2.98 \mathrm{e}-06$ \\
\hline 0.2 & 0.0891899350 & $3.64 \mathrm{e}-010$ & $2.58 \mathrm{e}-010$ & $2.47 \mathrm{e}-010$ & $3.94 \mathrm{e}-06$ & $2.02 \mathrm{e}-03$ & $5.46 \mathrm{e}-06$ \\
\hline 0.3 & 0.1176090956 & $3.99 \mathrm{e}-011$ & $5.59 \mathrm{e}-011$ & $4.56 \mathrm{e}-011$ & $5.85 \mathrm{e}-06$ & $1.52 \mathrm{e}-04$ & $7.33 \mathrm{e}-06$ \\
\hline 0.4 & 0.1347902526 & $1.29 \mathrm{e}-09$ & $8.77 \mathrm{e}-011$ & $9.64 \mathrm{e}-011$ & $7.70 \mathrm{e}-06$ & $2.20 \mathrm{e}-03$ & $8.50 \mathrm{e}-06$ \\
\hline 0.5 & 0.1405392142 & $2.04 \mathrm{e}-010$ & $1.38 \mathrm{e}-010$ & $1.66 \mathrm{e}-010$ & $9.47 \mathrm{e}-06$ & $3.01 \mathrm{e}-03$ & $8.89 \mathrm{e}-06$ \\
\hline 0.6 & 0.1347902526 & $1.29 \mathrm{e}-009$ & $8.77 \mathrm{e}-010$ & $9.64 \mathrm{e}-011$ & $1.11 \mathrm{e}-05$ & $2.20 \mathrm{e}-03$ & $8.50 \mathrm{e}-06$ \\
\hline 0.7 & 0.1176090956 & $1.60 \mathrm{e}-010$ & $5.59 \mathrm{e}-010$ & $4.56 \mathrm{e}-011$ & $1.26 \mathrm{e}-05$ & $1.52 \mathrm{e}-04$ & $7.33 \mathrm{e}-06$ \\
\hline 0.8 & 0.0891899350 & $3.64 \mathrm{e}-010$ & $2.58 \mathrm{e}-010$ & $2.47 \mathrm{e}-010$ & $1.35 \mathrm{e}-05$ & $2.02 \mathrm{e}-03$ & $5.46 \mathrm{e}-06$ \\
\hline 0.9 & 0.0498467900 & $1.24 \mathrm{e}-009$ & $5.87 \mathrm{e}-010$ & $5.77 \mathrm{e}-010$ & $1.20 \mathrm{e}-05$ & $2.68 \mathrm{e}-03$ & $2.98 \mathrm{e}-06$ \\
\hline
\end{tabular}

Table 7 : Comparison of solutions obtained by the present method for Bratu equation with other methods with $\lambda=2$ for example 6

\begin{tabular}{c|c|c|c|c|c|c|c}
\hline$x$ & $\begin{array}{c}\text { Exact } \\
\text { eigenvalues }\end{array}$ & $\begin{array}{c}\text { Our method } \\
n=10\end{array}$ & $\begin{array}{c}\text { Parametric } \\
\text { Spline [15] }\end{array}$ & $\begin{array}{c}\text { B-spline } \\
{[12]}\end{array}$ & $\begin{array}{c}\text { Nonpoly. } \\
\text { Spline } \\
{[13], N=10}\end{array}$ & $\begin{array}{c}\text { Laplace } \\
{[5]}\end{array}$ & $\begin{array}{c}\text { Decompo- } \\
\text { sition [11] }\end{array}$ \\
\hline 0.1 & 0.1144107440 & $1.44 \mathrm{e}-09$ & $1.25 \mathrm{e}-08$ & $2.98 \mathrm{e}-06$ & $9.71 \mathrm{e}-09$ & $1.98 \mathrm{e}-06$ & $2.68 \mathrm{e}-03$ \\
\hline 0.2 & 0.2064191156 & $1.98 \mathrm{e}-09$ & $1.95 \mathrm{e}-08$ & $5.46 \mathrm{e}-06$ & $1.41 \mathrm{e}-08$ & $3.94 \mathrm{e}-06$ & $2.02 \mathrm{e}-03$ \\
\hline 0.3 & 0.2738793116 & $1.18 \mathrm{e}-09$ & $2.73 \mathrm{e}-08$ & $7.33 \mathrm{e}-06$ & $1.98 \mathrm{e}-08$ & $5.85 \mathrm{e}-06$ & $1.52 \mathrm{e}-04$ \\
\hline 0.4 & 0.3150893646 & $9.84 \mathrm{e}-011$ & $3.31 \mathrm{e}-08$ & $8.50 \mathrm{e}-06$ & $2.42 \mathrm{e}-08$ & $7.70 \mathrm{e}-06$ & $2.20 \mathrm{e}-03$ \\
\hline 0.5 & 0.3289524214 & $6.03 \mathrm{e}-010$ & $3.53 \mathrm{e}-08$ & $8.89 \mathrm{e}-06$ & $2.60 \mathrm{e}-08$ & $9.47 \mathrm{e}-06$ & $3.01 \mathrm{e}-03$ \\
\hline 0.6 & 0.3150893646 & $9.84 \mathrm{e}-011$ & $3.31 \mathrm{e}-08$ & $8.50 \mathrm{e}-06$ & $2.42 \mathrm{e}-08$ & $1.11 \mathrm{e}-05$ & $2.20 \mathrm{e}-03$ \\
\hline 0.7 & 0.2738793116 & $1.18 \mathrm{e}-09$ & $2.73 \mathrm{e}-08$ & $7.33 \mathrm{e}-06$ & $1.98 \mathrm{e}-08$ & $1.26 \mathrm{e}-05$ & $1.52 \mathrm{e}-04$ \\
\hline 0.8 & 0.2064191156 & $1.98 \mathrm{e}-09$ & $1.95 \mathrm{e}-08$ & $5.46 \mathrm{e}-06$ & $1.41 \mathrm{e}-08$ & $1.35 \mathrm{e}-05$ & $2.02 \mathrm{e}-03$ \\
\hline 0.9 & 0.1144107440 & $1.44 \mathrm{e}-09$ & $1.25 \mathrm{e}-08$ & $2.98 \mathrm{e}-06$ & $9.71 \mathrm{e}-09$ & $1.20 \mathrm{e}-05$ & $2.68 \mathrm{e}-03$ \\
\hline
\end{tabular}

The maximum absolute errors in solutions of Bratu nonlinear problem are compared with methods in $[5,11,12,13,15]$ for $n=10$ and tabulated in Tables 6 and 7 . Table 6 shows that the absolute errors of the solutions for $\lambda=1$ are quite accurate and are in good agreement with the other methods. It is also noticed that for the case of $\lambda=2$ in Table 7 , the absolute errors in present method are reduced and are fast convergent than all other methods. Therefore, as the value of $\lambda$ increases the solutions are more accurate and reliable and our method is more efficient. The absolute errors of our method are depicted in Fig. 1 and 2, and are compared to the other methods.

\section{Conclusion}

In this study, the Spectral Collocation method is applied for solving linear and nonlinear second order eigenvalue problems, respectively. Besides, the present method is computationally efficient and much competent with the other published works earlier. Furthermore, this method with the aid of MATLAB code is well suited for both regular as well as singular Sturm-Liouville problems. Finally, the computational stable convergence for some eigenvalue problems is achieved. 


\section{Acknowledgments}

The first author is grateful to Dr. Samir Kumar Bhowmik, Department of Mathematics and Statistics, College of Science, Al Imam Mohammad Ibn Saud Islamic University, Riyadh, Kingdom of Saudi Arabia for his assistance in editing the article.

\section{REFERENCES}

[1] Bailey P., Everitt W. and Zettl. A., Computing eigenvalues of singular Sturm-Liouville problems, Results in Mathematics, 1-2 (1991), Vol. 20, pp. 391-423.

[2] Chen C.K and Ho S. H., Application of Differential Transformation to Eigenvalues problems, Applied Mathematics and Computation (1996), Vol.79, pp.173-188.

[3] Aregbesola A. S., Numerical solution of Bratu problem using the method of weighted residual, Electronic Journal of Southern African Mathematical Sciences (2003),Vol.3, pp. 1-7.

[4] Min M. S., and Gottileb D., Domain Decomposition Spectral approximation for an Eigenvalue problem with piecewise constant co-efficient, Mathematics of Computation, SIAM Journal of Numerical Analysis, Society for Industrial and Applied Mathematics, 1(2005), Vol. 43, pp. 502-520.

[5] Khuri S. A., A new approach to Bratu's problem, Applied Mathematics and Computation, 1 (2004), Vol. 147, pp.131-136.

[6] Chanane B., Computation of the eigenvalues of Sturm-Liouville Problems with parameter dependent boundary conditions using the Regularized Sampling Method, Mathematics of Computation, 252(2005),Vol. 74, pp. 1793-1801.

[7] Spectral Methods for Differential Problems, C. I. Gheorghiu."T. Popoviciu” Institute of Numerical Analysis, Cluj-Napoca, Romania, January 20, 2005-May 15, 2007.

[8] Celik I., Approximation of eigenvalues with the method of weighted residuals collocation method, Applied Mathematics and Computation, 2(2005),Vol. 160, pp. 401-410.

[9] Reutskiy S. Yu., The method of fundamental solutions for Helmholtz eigenvalue problems in simply and multiply connected domains, Engineering Analysis with Boundary Elements, 3(2006), Vol. 30, pp.150-159.

[10] Yucel U., Approximations of Sturm-Liouville eigenvalues using differential quadrature (DQ) method, Journal of Computational and Applied Mathematics, 2(2006), Vol.192, 310-319.

[11] Liao S., Tan Y., A general approach to obtain series solutions of nonlinear differential Equations. Studies in Applied Mathematics, 4(2007), Vol. 119, pp. 297-354.

[12] Caglar H., Caglar N., Özer M., Valaristos A., Anagnostopoulos A. N., B-spline method for solving Bratu's problem, International Journal of Computer Mathematics, 8(2010), Vol. 87, pp.1885-1891.

[13] Jalilian R., Non-polynomial Spline method for solving Bratu's problem, Computer Physics Communications, 11(2010), Vol.181, pp.1868-1872.

[14] Shen J. and Tang T., Spectral and High Order Methods with Application, Mathematics Monograph Series 3.

[15] Zarebnia1 M., Sarvari Z., Parametric Spline Method for Solving Bratu's Problem International Journal of Nonlinear Science, 1(2012), Vol.14, pp.3-10.

[16] Singh Randhir, Kumar Jitendra, Computation of Eigenvalues of Singular Sturm-Liouville Problems using Modified Adomian Decomposition Method, International Journal Nonlinear Science, 3(2013), Vol.15, pp.247-258.

[17] Atkinson, Kendall E, An Introduction to Numerical Analysis, John Wiley \& Sons, Ny, $2^{\text {nd }}$ edition, 1989. 
[18] Lui. S.H., Numerical analysis of partial differential equation, John Wiley \& Sons, Inc., Hoboken, New Jersey (2011).

[19] Taher A.H.S, Malek A., Masuleh S.H.M, "Chebychev differentiation matrices for efficient Computation of the eigenvalues of fourth order Sturm-Liouville problems," Applied Mathematical Modeling, 7(2013), Vol. 37, pp. 4634-4642.

[20] Shen Jie., Efficient Chebyshev-Legendre Galerkin methods for elliptic problems. In A. V. Ilin and R. Scott, editors, Proceedings of ICOSAHOM'95,(1996), pp. 233-240, Houston J. Math.

[21] Trefethen L. N., Spectral Methods in MATLAB. SIAM, Philadelphia, PA (2000). 